\title{
Studies of Seyfert galaxies in Fesenkov Astrophysical Institute
}

\author{
Denissyuk E. * Valiullin R.† Omarov CH. $;$ Shomshekova S. $\$$ Krugov M., and Omar B.॥ \\ Fesenkov Astrophysical Institute,Almaty, Kazakhstan
}

\begin{abstract}
This article provides an overview of the main research results of a large group of Seyfert galaxies, carried out at Fesenkov Astrophysical Institute (FAI) over the past 50 years. Spectral observations have been performed since 1970. At an early stage, a three cascade image-tube (UM-92) was used as a radiation receiver. In the $90 \mathrm{~s}$, the equipment was modernized, and at present, the modern CCD cameras are used as radiation detectors at the output of spectrographs.

The results of observations were used to determine the absolute fluxes of the emission lines and to study their profiles. Several additional emission features were detected on the wings of the broad emission lines $H \alpha$ in the spectra of two galaxies NGC 4151 and Ark 120. These features are emitted by compact ionized objects, rotating in the field of Central Body (CB).

Photometric observations of Seyfert galaxies have been carried out at FAI since 2010. Light curves of more than 20 Seyfert galaxies have been obtained. In particular, the light curves of the galaxy NGC 4151, obtained last years, shows that an active stage of its nucleus took place in 2015-2016, when the brightness increased by $0^{m} .5$ in the $\mathrm{V}$ filter, and by almost $2^{m}$ in the $\mathrm{R}$ filter. Then, in 2018 - 2019 there was a rather sharp decline of brightness, and B V R magnitudes returned to their minimal values.
\end{abstract}

Keywords:AGN, Seyfert galaxies, emission lines, individual objects: NGC 4151, Ark 120, $N G C$ 322\%, NGC 1068

\section{Introduction}

In 1968 a new diffraction spectrograph of an original design was developed in FAI. The threecascade image-tube UM-92, probably one of the most effective image amplifiers in the world, was used as a receiver Denissyuk (2003). The spectrograph was mounted to the telescope AZT-8 $(D=0.7 \mathrm{~m})$ and proofs the high efficiency for observations of the faint emission objects. A new spectrograph made it possible to obtain measurable spectra of the objects up to $18^{m}$ with the medium spectral resolution.

Regular spectral observations of the objects from the $1-s t$ Survey of the Markarian galaxies were carried out with the new spectrograph in $70-80 \mathrm{~s}$, sometimes together with the observers from SAO: V. Lipovetskii and V. Afanasiev. As a result of these observations, spectra of more than 300 galaxies from Markarian's lists were described, radial velocities were measured, and the absolute magnitudes of them were estimated Denissyuk (1971b), Denissyuk (1971c), Denissyuk (1971a), Denissyuk (1973), Denissyuk (1974a), Denissyuk (1974b) and Denissyuk (1974c). There were 49 Seyfert galaxies among them Denissyuk \& Lipovetskii (1973), Denissyuk \& Lipovetskii (1977) and Afanasiev et al. (1979). Currently observations of the galaxies are actively continued.

\footnotetext{
*eddenis@mail.ru, Corresponding author

${ }^{\dagger}$ rashit_valiullin@mail.ru

${ }^{\ddagger}$ chingis.omarov@gmail.com

${ }^{\S}$ shmshekva-saule@mail.ru

『mkrugov@astroclub.kz

" omar@aphi.kz
} 


\section{Observations and reduction}

The modern spectral observations are performed with the spectrographs, mounted to the eastern 1 meter telescope of Tyan Shan Observatory (TShAO) and to the telescope $A Z T-8$. CCD cameras SBIG STT-3200 $(2184 \times 1472,6.8 \mu)$ are the radiation receivers at the output of the spectrographs. The spectral range available for observation is $\sim 3500 \AA(4000-7500, \AA)$. During observations the spectra of the galaxy and standard star were obtained. The entrance slit of spectrograph about 5" - 10" is used, to ensure pass of the full fluxes as from the nucleus of galaxy and standard star. Obtained spectrograms were reduced in the standard manner: dark subtraction, flat fielding and wavelength calibration using $\mathrm{He}-\mathrm{Ne}-\mathrm{Ar}$ lines. All results were corrected for atmospheric extinction. Comparison of the observed energy distribution in the spectrum of the standard with the data from the Catalog Kharitonov et al. (2011) makes it possible to determine the spectral sensitivity of the equipment. After all reductions, the spectrum of a galaxy in the absolute energy units is obtained.

Photometric observations were carried out with the western 1 meter telescope (TShAO), equipped with the same CCD camera SBIG STT-3200 $(2184 \times 1472,6.8 \mu)$ and with the set of B V R filters. Field stars with the known brightness values were used as photometric standards. The primary image processing consists of standard operations with the Bias, Dark and Flat files. Image measurements are performed using standard IRAF and MaximDl 6 programs. The corresponding equations are used to transform the obtained instrumental brightness estimates to the standard system B V R.

\section{Results of spectral observations}

Search and analysis of emission details that are observed on the wings of broad hydrogen lines in the spectra of some galaxies is one of the aim of Seyfert galaxies study . The presence of such features was noted by a number of authors Boksenberg et al. (1975), Ulrich et al. (1991) and Shapovalova et al. (2009). So, in the very first spectrogram of the galaxy NGC 4151, published by Seyfert 9Seyfert (1943)), an additional emission was located on the red wing of the $H \beta$ line. Such emission features can be originated in the rather compact ionized bodies, while the center of the galaxy serves as the ionization source. It is possible to measure three parameters of the observable emission detailes: average velocity, total flux and width. Firstly it is necessary to subtract the wing of the broad emission line, on which this detail is superimposed. Only then it is possible to measure correctly the wavelength, flux and radial velocity of the emission detail. The radial velocities, measured relative to the center of the narrow emission line at different time reflect the motion of an object in the gravitational field of a supermassive CB.

Necessary observable data for time interval about 50 years $(1967$ - 2019) were obtained for the galaxies NGC 4151 and Ark 120. Based on these data, in the framework of classical mechanics, the orbits of motion of the bodies emitting such emissions were calculated, and the central masses of appropriate galaxies were estimated without taking into account the possible effects of general relativity.

\subsection{Seyfert galaxy NGC 4151}

Fragments of our spectrograms of the galaxy NGC 4151 in the red wavelengths range are presented in Fig. (1) Variations of the width and flux of the line with time are noticeable, and narrow emission features are distinct on the wing of the broad emission component. On the "blue" wing, a similar emission detail was observed only in a few spectrograms in 1997 and it disappeared in 2003. In order to separate emission feature in a pure form, the wing of the broad component was approximated by a high-order orthonormal polynomial, (in this case the 12th order) and was subtracted (see Fig. (2)). The result of subtracting is shown at the bottom of Fig. (2). The total width of this emission feature was about $\pm 1000 \mathrm{~km} / \mathrm{s}$. The results of processing of our spectrograms, obtained in $1997-2010$, allowed to construct the most probable orbit of the motion of the object, responsible for this additional emission, and, in particular, to estimate the mass of the CB of NGC 4151: $M=61-65 \times 10^{6} M_{\odot}$ Denissyuk et al. (2011). A weakening of the fluxes from this emission feature was noted as the object moved away from the ionization source Denissyuk et al. (2011). Since middle 2010, the shape of all emission 
lines in the spectrum of NGC 4151 remains unchanged, and additional emission details do not appear.

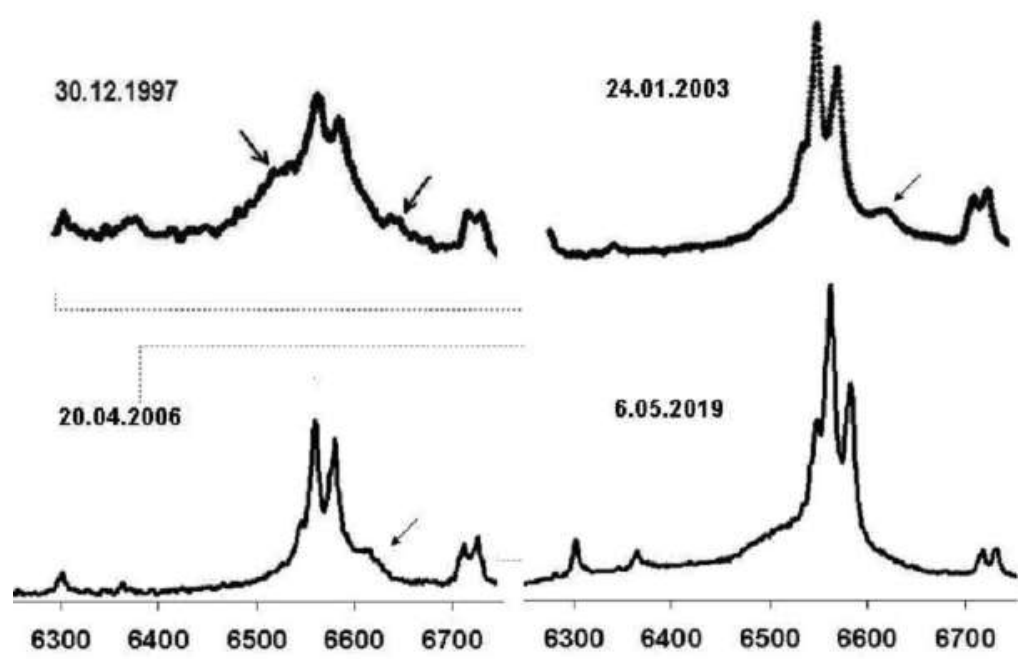

Figure 1. Fragments of spectrograms of the galaxy NGC4151 in the $H \alpha$ - line region. Emission features are indicated by arrows.

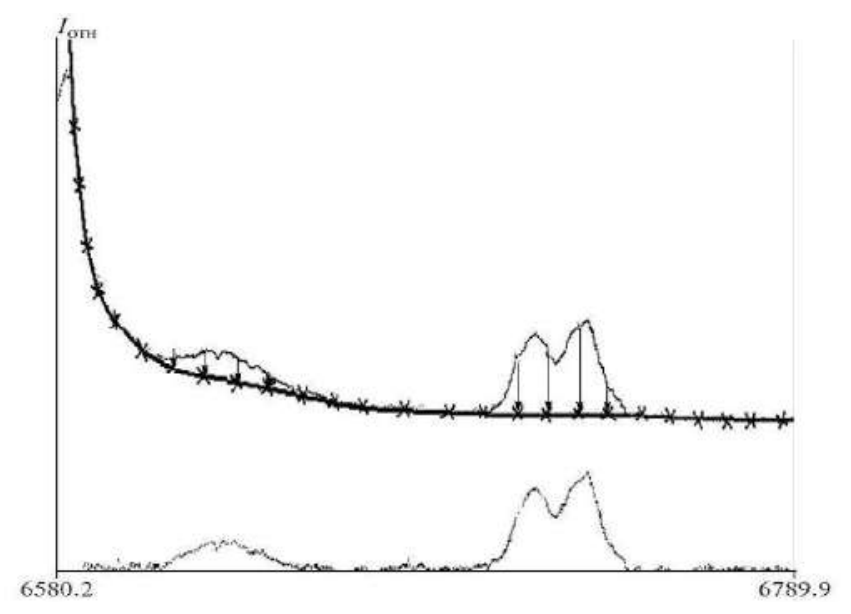

Figure 2. Scheme of subtraction of the broad wing of the $H \alpha$ - line. Bold line - is an orthonormal polynomial of the 12th order, approximating the "red" wing. The result of the subtraction is below.

\subsection{Seyfert galaxy Mrk $1095=$ Ark 120 .}

This rather bright galaxy was discovered by Arakelian Arakelian (1975) and was included in Catalog Markarian et al. (1989). It is actively explored in a wide range of wavelengths by both ground and space tools. The first spectrograms of this galaxy at FAI were obtained in November 1976. Subsequent observations of this galaxy revealed three emission details on the wings of the $H \alpha$ line, similar to those in the spectrum of NGC 4151. In this case, two objects emitting these emissions had positive radial velocities relative to the center of the narrow emission component, and the third object had a negative radial velocity. In addition to our data, we also used the results of observations, kindly provided by B. M. Peterson. After processing the observational data, the parameters of Keplerian orbits were obtained for three bodies emitting these emissions. The parameters of these three orbits are published in Denissyuk et al. (2015). A common parameter of these orbits is the central mass of this galaxy. It turned out to be equal $(167.5 \pm 2.8) \times 10^{6} M_{\odot}$. Fig. (3) shows the radial velocities of these three 
objects, measured relative to the center as a function of time. As in the case of the galaxy NGC 4151, the removal of such objects from the pericenter leads to a noticeable attenuation of their radiation. Fig. (4) shows two spectrograms of Ark 120, obtained in middle of December 2014 and in 2015 with an exposure of 1.5 hours. Two emission features with the radial velocities of -2160 and $+2510 \mathrm{~km} / \mathrm{s}$ are marked by arrows on the spectrogram of 2014, and those with the velocities $-3030,+1690$ and $+2270 \mathrm{~km} / \mathrm{s}$ are marked on the spectrogram of 2015 Fig. (3). As the intensities of these emissions are small, some of them may be a noise.

It is clear that spectral observations of this and other Seyfert galaxies should be continued, highly desirable in cooperation with other observatories. The probability is high that the nucleus of Ark 120 is binary Yan-Rong et al. (2019). This result was obtained, in particular, on the basis of observations made at FAI. Spectral observations of this and other Seyfert galaxies continue in order to search for the new emission details.

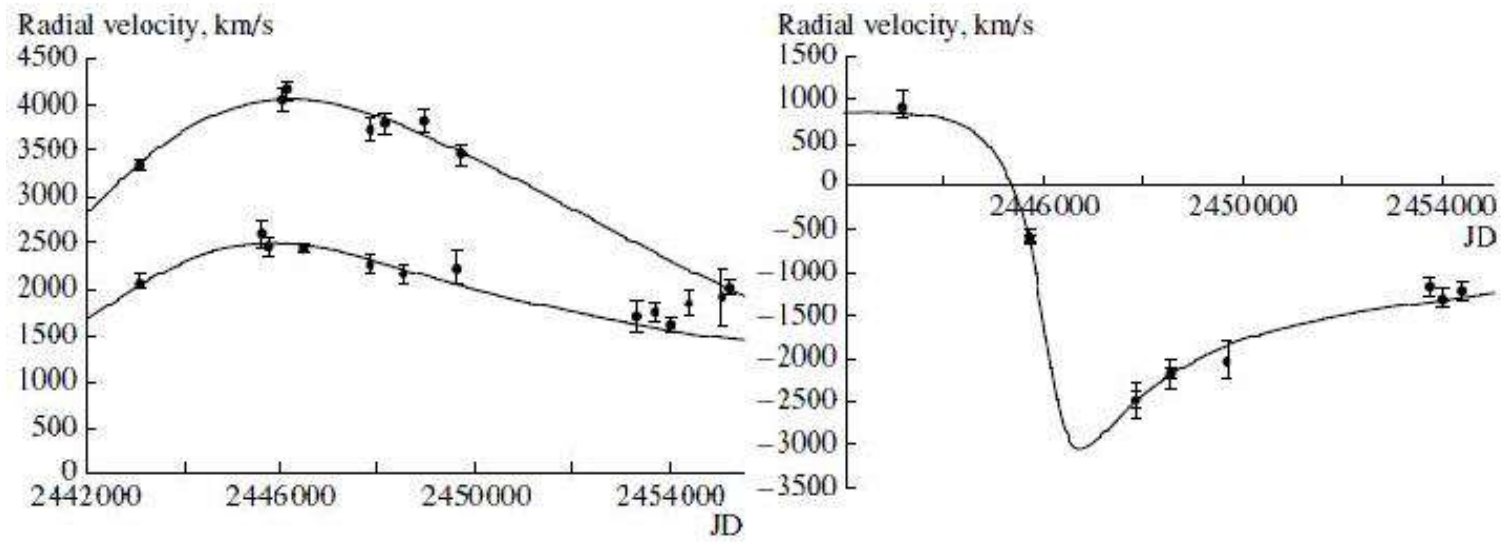

Figure 3. Radial velocities of the three emission features relative to the centre of the galaxy Ark 120 in dependence on the time.
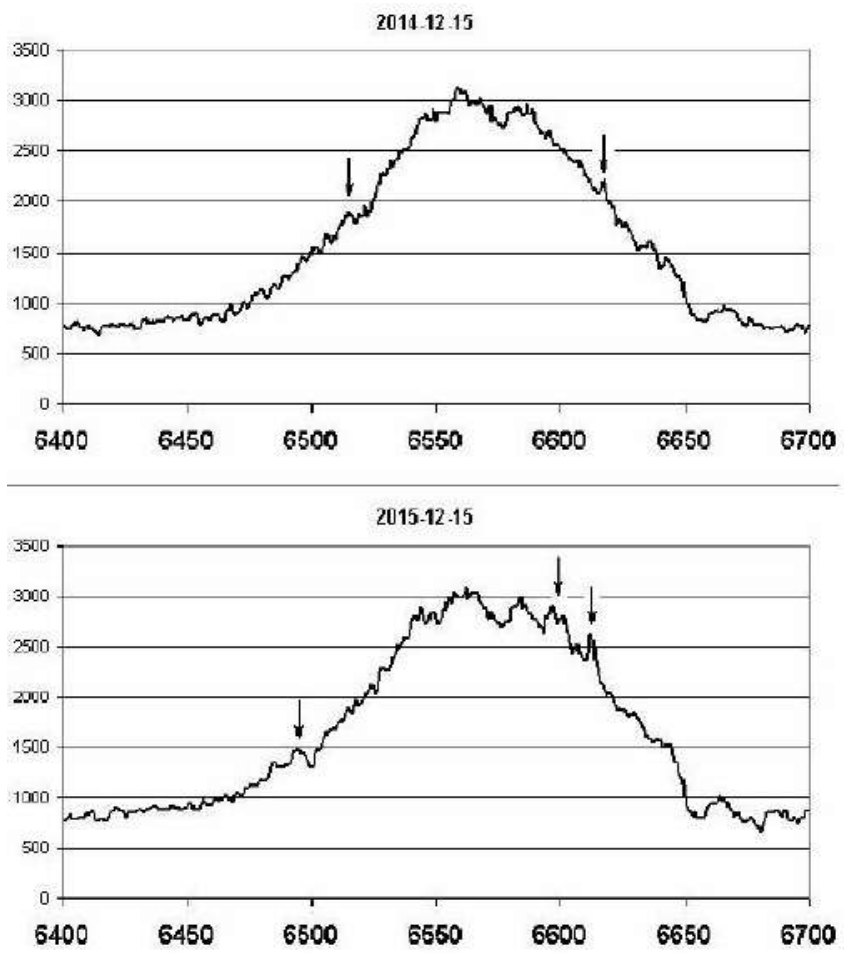

Figure 4. Emission features on the wings of $H \alpha$ in 2014 and 2015 in the spectrum of the galaxy Ark 120. 


\subsection{Galaxy NGC 1068}

Galaxy NGC 1068 belongs to the class Sy2, it is a fairly bright object $\left(V=8^{m} .9\right)$ and one of the closest to the Sun galaxies. In the spectrum of this galaxy, broad components of the hydrogen lines are practically absent. Fig. (5) (upper panel) shows a fragment of the original spectrogram in the region of the $H \alpha$ line, obtained using the AZT- 8 telescope with an exposure of 1.5 hours. Profiles of the $H \alpha,[\mathrm{NII}], 6548,6583 \AA$ emission lines in the center and on the 10 " distance on both sides of the continuum are presented on bottom panel. It can be seen that the position of these lines on the spectrogram varies depending on the distance to continuum. This is the result of rotation of emission objects at different distances from the center of galaxy. The results of measurements of the positions of two emission lines at different distances from the center on both sides of the continuum made it possible to construct rotation curve of the galaxy. Fig. (6) shows the differences in the rotation of the eastern and western (relative to the center) parts of the galaxy. In the region of $\pm 1.5 \mathrm{kpc}$, the rotation is close to solid-state. If we assume that at a distance of $6 \mathrm{kpc}$ the average observed velocity of rotation is about $300 \mathrm{~km} / \mathrm{s}$ and take into account the inclination of the galactic plane to the line of sight, then the mass inside this radius can be estimated using the formula $M_{\odot}=V^{2} r / G$. Then the mass will be equal to approximately $8 \times 10^{11} M_{\odot}$.

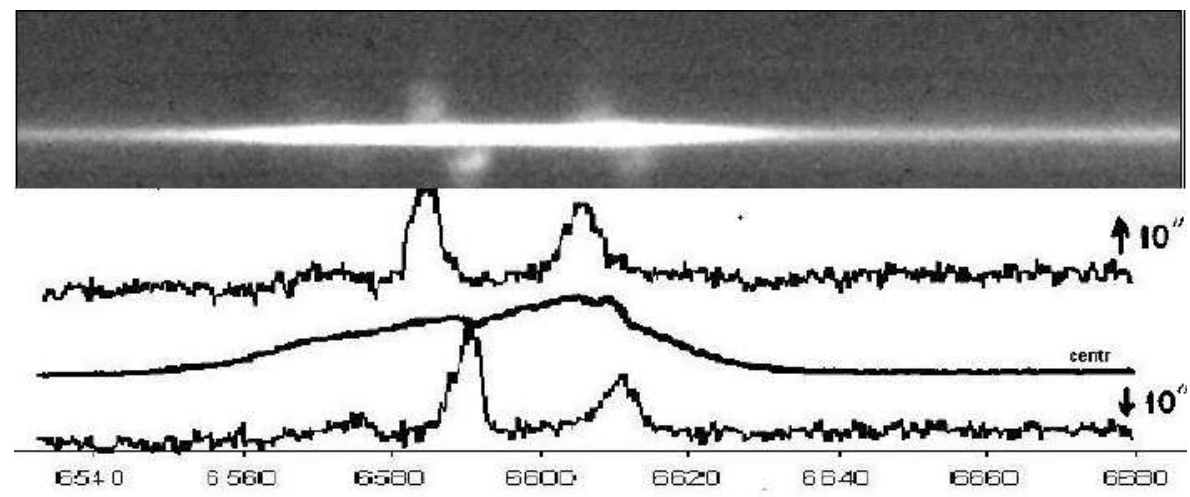

Figure 5. Fragment of the spectrogram of the galaxy NGC 1068 with the $H \alpha$ and [NII] emission lines (upper panel). Bottom panel-emission profiles of $H \alpha$, [NII], 6548, 6583 $\AA$ along continuum and on the $10^{\prime \prime}$ distance on both sides of it. Axis X- wavelengths in angstroms, axis Y-intensities in arbitrary scale.

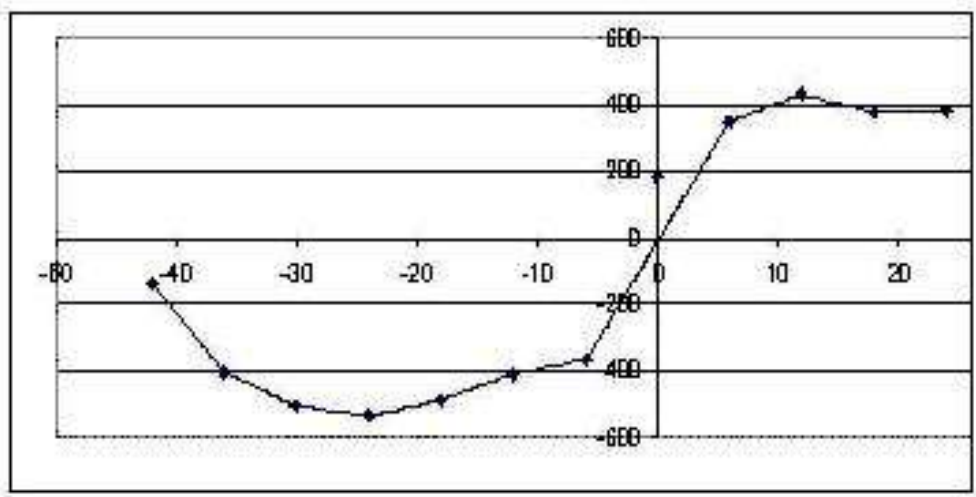

Figure 6. Rotation curve of the galaxy NGC 1068 from Denissyuk \& Valiullin (2019). Axis X-distance the centre in arcsec, axis Y-radial velocity in $k m=s e c$.

\subsection{Results of photometric observations}

Photometric data for more than 20 Seyfert galaxies were obtained in FAI during the last 10-12 years Shomshekova et al. (2019). The light curves of the galaxy NGC 4151, obtained last years are 
presented in Fig. (7). An active stage of nucleus of this galaxy was observed in 2015 - 2016, the brightness in the $\mathrm{V}$ filter increased by $0^{m} .5$, and in the $\mathrm{R}$ filter by almost $2^{m}$. Then, in $2018-2019$ there was a rather sharp decline of brightness, and B V R magnitudes returned to their minimal values. The radiation intensity of the emission feature depends on strength of the ionizing source and on the distance to it. The delay in the reaction of the emission feature to a change in the brightness of the $\mathrm{CB}$ reflects the absolute distance of this object from the nucleus, and may be a criterion for checking the orbit parameters.

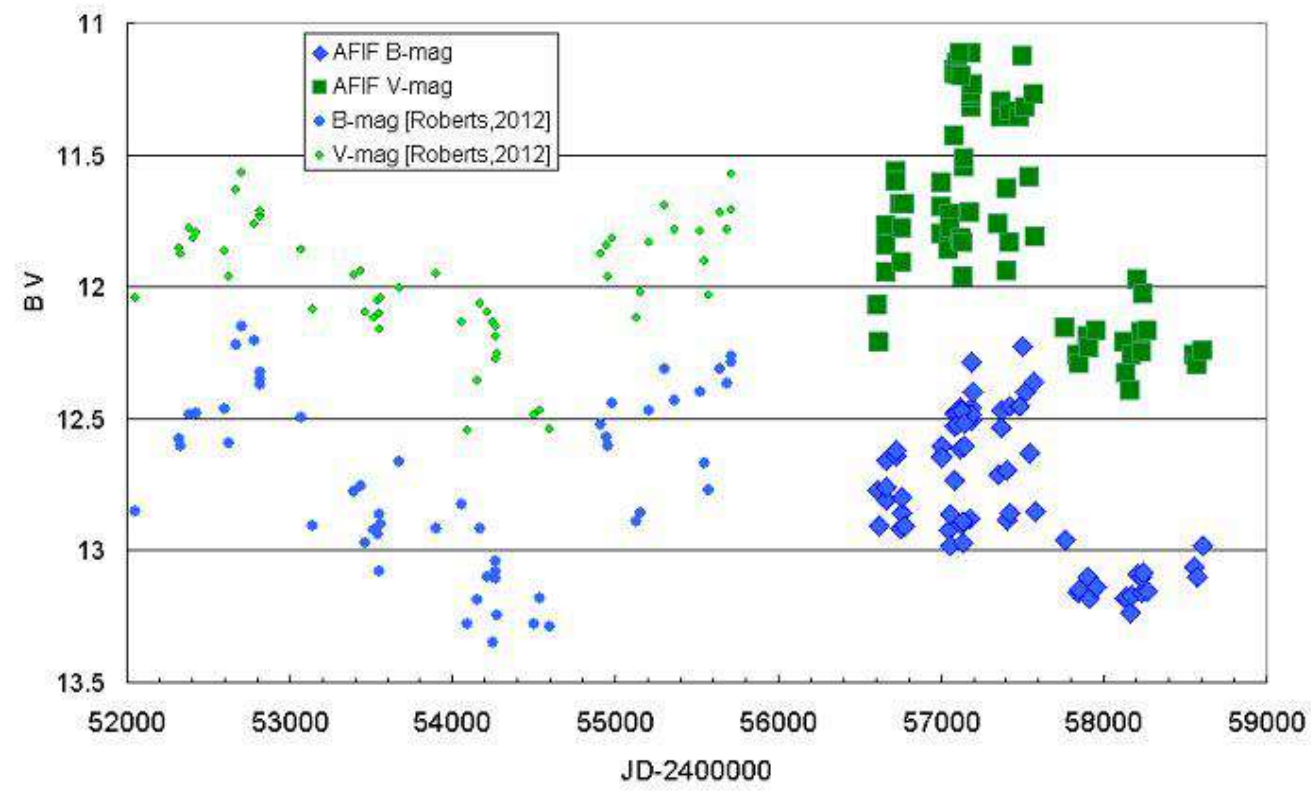

Figure 7. Light curves of the galaxy NGC 4151. Axis X -JulianDates, axis Y - B and V magnitudes.

\section{Conclusions}

Spectral observations of Seyfert galaxies unambiguously indicate the appearance in the vicinity of the Center of objects emitting sufficiently powerful emission lines in the presence of strong ionizing radiation. Such emission details are also observed on the wings of broad emission lines in the far ultraviolet.

The power of these emissions is a significant part, up to 10 percent, of the power of the broad emission component. There are no reliable estimates for the mass of matter emitting the broad component. However, it is obvious that this mass should be several orders of magnitude larger than the solar mass. Accordingly, the mass of objects, emitting emission features, should be at least ten solar masses, and possibly much more. Emission features have a noticeable width, caused to rotation, most likely. It is highly probable that such objects do not have their own energy sources and are invisible at a large distance from the center of the galaxy.

The rather frequent appearance of such objects near the galactic center may indicate that they are as ordinary members of the galaxy as stars, nebulae, or interstellar medium.

Taking part in the general rotation, some of such objects, can change their orbit due to random interactions with other masses, and appear in the Center where they are ionized and become visible. The spatial orbits of such objects, built on the basis of the dependence of their radial velocities on time, can be checked independently. To do this, it is enough to measure the time delay of their intensity changes from fluctuations in the brightness of the ionizing CB. It is important to have regular data on the dependence of the brightness of galactic CB on time. For a number of galaxies, similar observations are also carried out in FAI. 


\section{Acknowledgements}

This work was supported by the funding program BR05236322 of the Ministry of Education and Science of the Republic of Kazakhstan

\section{References}

Afanasiev E., Denissyuk E., Lipovetskii V., 1979, Pisma v Astronomicheskii Zhurnal, p. 271

Arakelian M., 1975, SoByu, 3, 47

Boksenberg P., Shortridge K., Allen D., et al. 1975, MNRAS, 173, 381

Denissyuk E., 1971a, Astronomicheskii Circular, 1, 624

Denissyuk E., 1971b, Astronomicheskii Circular, 4, 614

Denissyuk E., 1971c, Astronomicheskii Circular, 7, 621

Denissyuk E., 1973, Astronomicheskii Circular, 2, 759

Denissyuk E., 1974a, Astronomicheskii Circular, 1, 809

Denissyuk E., 1974b, Astronomicheskii Circular, 2, 809

Denissyuk E., 1974c, Astronomicheskii Circular, 2, 837

Denissyuk E., 2003, Astron. Astroph. Transactions, 22, 175

Denissyuk E., Lipovetskii V., 1973, Astronomicheskii Circular, 1, 798

Denissyuk E., Lipovetskii V., 1977, Pisma v Astronomicheskii Zhurnal, p. 7

Denissyuk E., Valiullin R., 2019, News of National. Acad. of Sci. Republic Kazakhstan, 3, 153

Denissyuk E., Valiullin R., Gaisina V., 2011, Astronomicheskii Zhurnal, 9, 1, 88

Denissyuk E., Valiullin R., Gaisina V., 2015, Astronomy Reports, 59,2, 123

Kharitonov A., Tereschenko V., Knyazeva L., 2011, Spectrophotometric Catalog of stars, Almaty

Markarian B., Lipovetsky V. ans Stepanian J., et al. 1989, Soobshch. Spets. Astrof. Obs., 62, 5

Seyfert C., 1943, ApJ, 28, 97

Shapovalova A., Popovic L., Burenkov A. N., 2009, arXiv:0910.2980v1

Shomshekova S., Denissyuk E., Valiullin R., et. al. 2019, Astrophysics, 62, 2, 187

Ulrich M. H., Boksenberg A., Penston M., et al. 1991, ApJ, 483, 382

Yan-Rong L., Jian-Min W., Zhi-Xiang Z., et. al. 2019, Astroph. J., 331, 241 